\title{
MR imaging features and contrast enhancement characteristics in benign and malignant breast lesions
}

Hoon T. S. , Ansari M. A., Shuaib I. L., Ariff A. R. Mohd., Khalid I., Basheer MB. L. Mohd., Tun M., Aziz Mohd. E.

Dept. of Radiology, Hospital Universiti Sains Malaysia (HUSM), Health Campus, Universiti Sains Malaysia, Kubang Kerian, Kelantan, Malaysia.

Advanced Medical and Dental Institute, Universiti Sains Malaysia, Pulau Pinang, Malaysia.

Dept. of Radiology and Imaging, Tribhuvan University, Teaching Hospital, Maharajgunj, Kathmandu, Nepal.

Correspondence address: Dr. Mukhtar Alam Ansari, Dept. of Radiology and Imaging, Tribhuvan University, Teaching Hospital, Maharajgunj, Kathmandu, Nepal

Email: dr.mukhtaransari@gmail.com

\begin{abstract}
Introduction: Breast cancer is the commonest female malignancy in Malaysia and other countries the world. All races are affected in Malaysia and breast cancer comprised $30.4 \%$ of all newly diagnosed cancer cases. A women in Malaysia has a 1 in 19 chances of getting breast cancer in her lifetime. The Age Standardized Rate (ASR) of female breast cancer in Malaysia is 52.8 per 100,000 people and is higher than that in Singapore, Hong Kong and Shanghai, but it is lower than that in Australia and the UK. Out of the 4,337 new cases of female breast cancer cases reported to the National Cancer Registry of Malaysia in 2002, 52.3\% were for women less than 50 years old. Genetic risk factors have not been studies thoroughly in Malaysia.

Methods: We prospectively studied 55 patients with breast lumps in whom MR imaging was performed. The T1- and T2-weighted, axial STIR, fat-suppression contrast enhanced fast spin echo and two dimensional dynamic enhanced fast spoiled gradient-echo images were obtained. The tumour margins and shape, enhancement pattern and time-signal intensity curves were analysed.

Results: A total of 37 patients fulfilled the study criteria with the mean age of $43.46 \pm 11.99$ years (age ranged 21-70). There were 23 benign and 14 malignant lesions. The MR imaging criteria suggestive of malignancy were poorly defined and spiculated margins, irregular shape, heterogeneous and rim enhancement as well as type II and III curves. On the other hand, the criteria for a benign breast lesions were well-defined margin, regular and lobulated shape, none or homogeneous enhancement and type I curve. Only the malignant lesions are characterised by skin, retroareolar, nipple and pectoralis muscle involvement.
\end{abstract}

Conclusion: MR imaging is a valuable complementary breast imaging tool to further evaluate equivocal findings on conventional $\mathrm{x}$-ray mammography. A combination of lesion morphology and enhancement characteristics is useful in distinguishing benign from malignant lesions.

Keywords: MRI, mammography, benign, malignant, breast

\section{Introduction}

Breast cancer is the commonest female malignancy in Malaysia and other countries the world. All races are affected in Malaysia and breast cancer comprised 30.4\% of all newly diagnosed cancer cases. A women in Malaysia has a 1 in 19 chances of getting breast cancer in her lifetime. The Age Standardized Rate (ASR) of female breast cancer in Malaysia is 52.8 per 100,000 people and is higher than that in Singapore, Hong Kong and Shanghai, but it is lower than that in Australia and the UK. Out of the 4,337 new 
cases of female breast cancer cases reported to the National Cancer Registry of Malaysia in 2002, 52.3\% were for women less than 50 years old. Genetic risk factors have not been studies thoroughly in Malaysia. Women in Malaysia present with larger tumors and at later stages than women in the US. Certain health beliefs, lack of correct information, and inadequate health care facilities are a barrier to routine early detection and treatment of the disease. There is in addition no mass screening program for breast cancer in Malaysia.

At present, conventional x-ray mammoraphy and clinical breast examination are the current standard measures for breast cancer screening and initial evaluation of breast signs and symptoms. The combination of conventional $\mathrm{x}$-ray mammography and clinical breast examination has a moderate sensitivity and high specificity for breast cancer. Conventional x-ray mammography, which is readily available, relatively inexpensive and suited for microcalcification detection, remains the primary imaging modality for breast evaluation. However, detection of lesions at conventional x-ray mammography depends on many factors: the technical quality of the mammograms, the contrast between the lesions and adjacent normal breast parenchyma and the experience of the readers. Approximately $5-15 \%$ of palpable breast cancers are not detected with mammography. This means some cancers goes undetected at conventional x-ray mammography in early stage resulting in delay in diagnosis and treatment. Despite several technical advances affecting the actual data acquisition as well as film processing, several limitations of mammography remains well documented. These includes difficulties in assessing dense glandular breast tissue, regions located close to the chest wall or within axilla,1 breast implants and post operative changes.

Mammography tends to underestimate tumour size and multifocality. Mammography and ultrasonography significantly underestimated tumour size by $14 \%$ and $18 \%$ respectively 2. Multicentricity of breast cancer has been reported at MR imaging in a substantial number of patients whose mammograms otherwise showed unifocal disease 3, 4, 5. These limitations have stimulated exploration into alternative or adjunctive imaging modalities to improve lesion detection and characterisation.

MR imaging of the breast is emerging as a very powerful technique for detection, diagnosis and monitoring of breast abnormalities. It provides better soft tissue contrast resolution than conventional $\mathrm{x}$-ray mammography and does not require ionizing radiation. It has multiplanar imaging capability compared to the other imaging modalities. Hence, this improves lesion detection and characterisation. With improvements of breast surface coils and pulse sequences designs, as well as the increased use of gadolinium based contrast agents, breast MR imaging has rapidly evolved in recent years. The early success of contrast enhanced MRI has lead to considerable enthusiasm about its potential clinical impact. In particular, it has been suggested that MRI may be helpful in further evaluating patients that have abnormal mammograms, especially for the women with breast cancers who desire breast conservation therapy than mastectomy.

\section{Methods}

This prospective study was carried out in the Dept. of Radiology, Hospital Universiti Sains Malaysia (HUSM), Health Campus, Universiti Sains Malaysia, Kubang Kerian, Kelantan, Malaysia. A total of 55 patients who presented with breast lump were included in the study for MR imaging examination of the breast.

Inclusion criteria

1. Patients of all age and ethnic groups.

2. No contraindication to MR examination.

3. Cytology/histopathologically confirmed benign or malignant breast diseases.

4. Patient consented for examination.

Exclusion criteria

1. Patient with pregnancy.

Out of 55 patients 18 were excluded from the study due to lack of cytology/histopathology confirmation results.

MR imaging protocol

MR imaging was performed using 1.0-Tesla whole body MR system (GE Signa Horizon LX, Milwaukee, US) using a circular-polarised body coil for RF transmission and dedicated double-breast coil for RF detection. All patients were imaged in the prone position. No compression device was used. An intravenous canula was inserted prior to the examination. It was connected to the long tube to allow the injection of contrast medium from outside the bore of the magnet. After the acquisition of a precontrast scan, gadopentetate dimeglumine (Magnevist; Schering) was injected manually as a bolus dose of $0.10 \mathrm{mmol} / \mathrm{kg}$ followed by $10 \mathrm{ml}$ of saline flush. During the injection dynamic scan was acquired.

MR imaging sequences were performed based on our department protocols. There were sagittal T1-weighted fast spin-echo (TR/TE, 500-600/10-15 msec), sagittal T2weighted fast spin-echo (TR/TE, 3600-4300/99-101 msec), 
axial T1-weighted fast spin-echo (TR/TE, 500-600/10-15 msec), axial STIR (TR/TE, 4000-6000/12-13 msec; TI, 140-150 msec), two-dimensional, dynamic enhanced, fast spoiled gradient-echo (FSPGR TR/TE, 150/3.0-4.0 msec; flip angle, 90 degree) and contrast-enhanced axial and sagittal T1 weighted fat suppression fast spin-echo. The dynamic series lasted for six minutes. This consisted of dynamic images before and after the rapid bolus of intravenous gadopentetate dimeglumine injection.

All MR imaging studies were assessed by a radiologist with knowledge of clinical findings but blinded to the cytology/ histopathology results. The number, border (well-defined, ill-defined, spiculated), shape (regular, irregular, lobulated), size (maximal diameter) and enhancement pattern (homogenous, heterogeneous, rim) of all the lesions were evaluated. The signal-time curve was obtained in suspicious breast lesions using the 'Functool' program installed in the Advantage Window workstation (GE Medical Systems, US). It was to depict the lesions' enhancement behaviour in the early, intermediate and late post-contrast periods. The signal-time curves were then classified as steady (type I), plateau (II) or washout (type III) as shown in Figure 1.6

Type I - straight or curved.

There is a monotonic increase in signal intensity during a 6 minutes examination.

Type II - plateau.

There is an initial upstroke, after which enhancement is abruptly cut off and the signal intensity plateaus in the intermediate and late postcontrast period.

Type III - washout.

There is an initial upstroke, after which enhancement is abruptly cut off and the signal intensity decreases in the intermediate postcontrast period.

Signal intensity

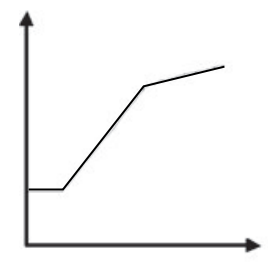

Type I (steady)

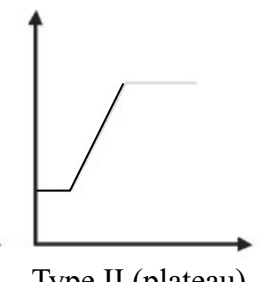

Type II (plateau)

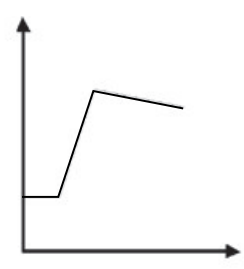

Type III (washout)
Fig. 1. Schematic drawing of time-signal intensity curves in contrast enhancement kinetics and their classification.

The study design and protocol were reviewed and approved by the USM Health campus research and ethical committee.

\section{Statistical Analysis}

Statistical analysis was done using SPSS for Windows version 9.0 software and Intercooled Stata 7.0 program. The sociodemographic data was analysed using descriptive analyses. The student $t$ test for independent samples was applied to check for a statistically significant difference between the age group and size of benign and malignant lesions. In univariate analysis, the associations between the various MR imaging parameters and time-signal intensity curve type and cytology/histopathology were determined. The chi-square test was used for the univiarate analysis. For all the tests, a P-value of less than 0.05 was taken as significant level. The significant parameters were analysed for sensitivity, specificity, positive predictive value, negative predictive value and overall accuracy. The null hypotheses were: there is no correlation between the MR features of breast lesions and cytology/histopathology results and there is no correlation between the enhancement characteristics and cytology/histopathology results.

\section{Results}

A total of 37 patients included in the study, 23 had benign and 14 had malignant breast lesions. The mean age for patients with benign and malignant groups was $40.26 \pm 10.31$ years and $48.71 \pm 13.1$ years, respectively. Independent $t$ test revealed no statistical significance in the mean age for these two groups (p-value of 0.298). When age distribution was further subgrouped into below and above 50 years of age, $82.6 \%$ of patients with benign breast lesions were below 50 years age. Four patients were 50 years and above, whereas for the patients with malignant lesions, they were equally distributed in these two age groups. Three patients $(8.1 \%)$ on hormone replacement therapy were accounted in the study. The duration of hormone replacement therapy was one to six years. Two had benign and one had malignant breast lesions. Analysis based on chi square test showed there was no statistically significant correlation between the family history of breast cancer or hormone replacement therapy and cytology/histopathology results. Table-1. showed the lesions detected on MR imaging, 23 (62.2\%) were benign and $14(37.8 \%)$ malignant. Of these benign breast lesions, 12 fibroadenomas, 6 fibrocystic changes and 5 were breast cysts. The malignant lesions were mainly infiltrating ductal carcinomas. Only one aprocine carcinoma encountered. No DCIS was found in this study. 
Table 1: Breast lesions.

\begin{tabular}{|c|c|c|c|}
\hline & & Frequency & Total \\
\hline \multirow{3}{*}{ Benign } & Fibroadenoma & $12(52.2 \%)$ & \multirow{4}{*}{23} \\
\hline & Fibrocystic changes & $6(26.1 \%)$ & \\
\hline & Cyst & $5(21.7 \%)$ & \\
\hline \multirow{3}{*}{ Malignant } & $\begin{array}{l}\text { Infiltrating ductal } \\
\text { carcinoma }\end{array}$ & $13(92.9 \%)$ & \\
\hline & & & \multirow[t]{2}{*}{14} \\
\hline & Apocrine carcinoma & $1(7.1 \%)$ & \\
\hline
\end{tabular}

All the diagnosis were proven by cytological examination. Only nine of them were then reconfirmed by histopathology.

Majority of malignant lesions were found in the upper outer quadrant and retroareolar region which account for $35.7 \%$. This was followed by upper inner $(21.4 \%)$ and lower outer (7.1\%) quadrant. For benign lesions, the commonest site was upper outer quadrant with least common site in the lower inner quadrant. Majority of the malignant lesions exhibited either spiculated $(57.1 \%)$ or poorly defined (35.7\%) margins (Fig. 2).

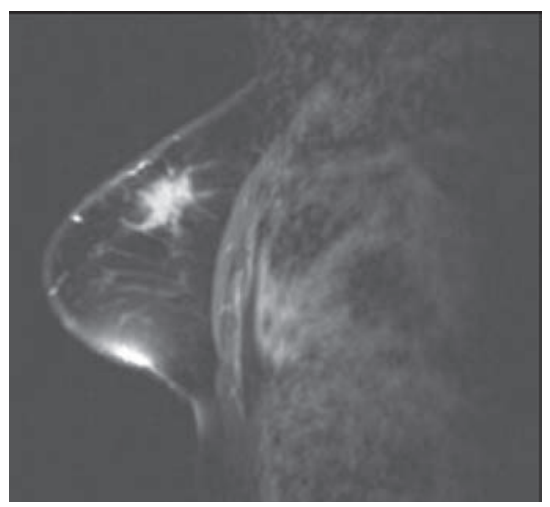

Fig. 2: Contrast-enhanced MR image showing a spiculated breast lesion.

Only 1 lesion demonstrated well-defined border. Most of them were irregular in shape. All these lesions were of low signal intensity on T1-weighted image. The signal intensity on T2WI were variable, 9 of the 14 lesions were highly intense while the others were of low signal intensity. However all these lesions were highlighted on STIR sequences. Thirteen lesions $(92.9 \%)$ characterised by heterogeneous enhancement pattern following intravenous contrast administration whereas the other lesion demonstrated rim enhancement pattern.

All benign lesions were characterised by well-defined border (Fig. 3).

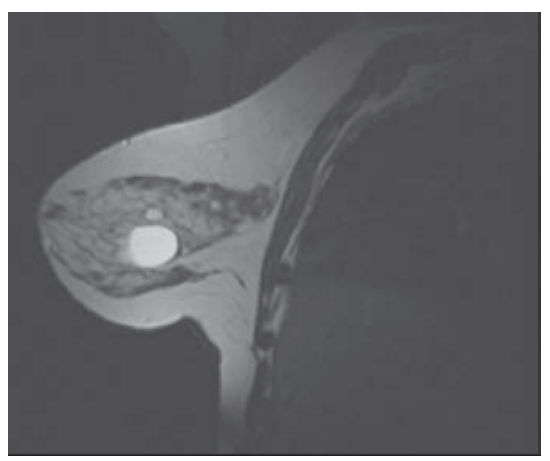

Fig. 3: T1-weighted MR image showing a well-defined breast lesion.

They were either regular $(73.9 \%)$ or lobulated $(26.1 \%)$ in shape. None of them had irregular outline. They were of low signal intensity on T1-weighted images and $78.3 \%$ were highly intense on T2-weighted images. Four lesions were isointense while one was hypointense to the fibroglandular tissue on T2-weighted images. Like malignant lesions, they were highlighted on STIR sequences.

Homogenous enhancement pattern was seen in 16 out of 23 lesions (Fig. 4). 


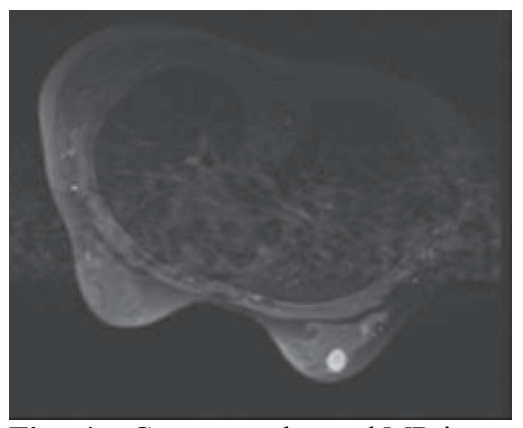

Fig. 4: Contrast-enhanced MR image showing a homogenous enhancing breast lesion.

Six lesions were not enhanced and only one had heterogeneous enhancement pattern. The non-enhancing lesions were confirmed as fibrocystic changes and breast cysts while the heterogeneous enhancing lesion were proven breast cyst with highly dysplatic cells. None of the benign lesions showed rim enhancement pattern. There were three lesions where dynamic study was not performed. Hence, only time-signal intensity curve of 34 lesions were evaluated. The shape of the time-signal intensity curves of benign and malignant lesions differed significantly. (Table. 2)

Table 2: Correlation between type of time-signal intensity curve and breast lesions.

$\begin{array}{cccc}\begin{array}{c}\text { Time-signal } \\ \text { intensity curve }\end{array} & \text { Benign } & \text { Malignant } & \text { Total } \\ \text { No enhancement } & 4(20.0 \%) & 0 & 4 \\ \text { Type I } & 16(80.0 \%) & 1(7.1 \%) & 17 \\ \text { Type II } & 0 & 2(14.3 \%) & 2 \\ \text { Type III } & 0 & 11(78.6 \%) & 11 \\ \text { Total } & 20 & 14 & 34\end{array}$

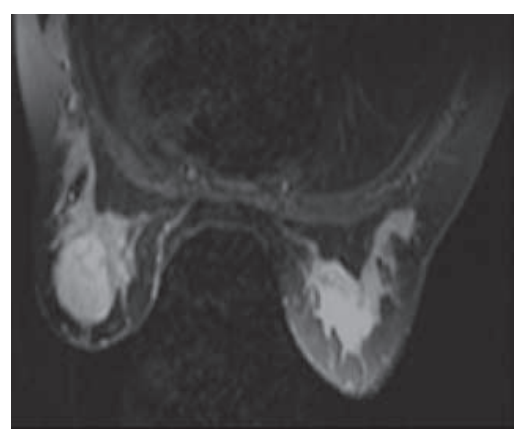

Fig. 5: Contrast-enhanced MR image showing a mass with internal septation.
Neither malignant nor other benign lesions demonstrated internal septation. Similar to the necrosis, the involvement of skin, retroareolar, nipple (Fig. 6) and pectoralis muscle were demonstrated exclusively in the malignant lesions only. Therefore, presence of these structures were suggestive of a malignant breast lesion. All cytology/histopathology proven benign lesions did not establish these features.

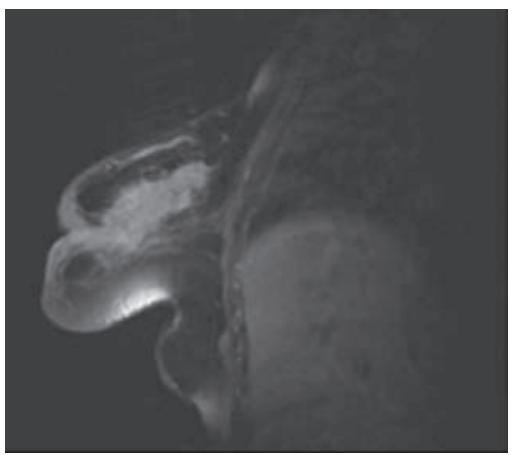

Fig. 6: Contrast-enhanced MR

image showing nipple retraction and thickened skin.

Using cytological/histopathological examination as the gold standard, the sensitivity, specificity, predictive value and accuracy of border, shape, enhancement patterns and time-signal intensity curves were analysed.

The MR imaging criteria suggestive of malignancy were poorly defined and spiculated margin, irregular shape, heterogeneous and rim enhancement as well as type II and III curves. On the other hand, the criteria for a benign breast lesion were well-defined margin, regular and lobulated shape, no or homogenous enhancement and type I curve. Hence the following diagnostic indices were derived for each individual parameter.

Among the evaluated variables, the feature with highest sensitivity was enhancement pattern $(100 \%)$ and the lowest sensitivity was the shape of the mass $(85.7 \%)$. The specificity was $100 \%$ for all the criteria except enhancement pattern.

The spiculated margin, irregular shape and type II and III curves were characteristic of a malignant lesion with PPV of $100 \%$. Well-defined margin, regular and lobulated shape, no or homogenous enhancement and type I curve were highly predictive of benign disease. The NPV were $95.8 \% ; 92.0 \% ; 100 \%$ and $95.2 \%$ respectively. Overall accuracy based on the tumour margin, shape, enhancement pattern and time-signal curve was $97.3 \%, 94.6 \%, 97.3 \%$ and $97.0 \%$ respectively (Table. 3 ). 
Table 3: Diagnostic indices for MR parameters.

\begin{tabular}{|c|c|c|c|c|c|}
\hline Parameters & Sensitivity (\%) & Specificity (\%) & PPV (\%) & NPV (\%) & Accuracy (\%) \\
\hline Border & 92.8 & 100 & 100 & 95.8 & 97.3 \\
\hline Shape & 85.7 & 100 & 100 & 92.0 & 94.6 \\
\hline $\begin{array}{c}\text { Enhancement } \\
\text { pattern }\end{array}$ & 100 & 95.6 & 93.3 & 100 & 97.3 \\
\hline $\begin{array}{l}\text { Time intensity } \\
\text { curve }\end{array}$ & 92.8 & 100 & 100 & 95.2 & 97.0 \\
\hline
\end{tabular}

\section{Discussion}

Sixty percent of breast cancers are found in the upper and outer quadrant of breast. This is followed by upper inner, lower outer and lower inner quadrant. Similar findings were also found in our study whereby benign and malignant lesions involved upper outer quadrant most commonly. In this study, the margin, shape and size of the lesions were analysed. Generally benign lesions were well-defined and regular in outline. They were smaller in size as compared to malignant lesions. In contrast, malignant lesions revealed either poorly defined or spiculated margin and irregular shape. These findings support several previous studies. Orel et al 7 identified fifteen of sixteen carcinomas exhibited at least partially irregular borders. Nunes et al reported absence of a lesion at MR imaging, smooth masses, lobulated masses with non-enhancing internal septation, and lobulated masses with minimal or no enhancement had high negative predictive value. Enhancing irregular masses and enhancing spiculated masses without septation were almost always malignant $8,9,10,11$. We observed presence of internal septation in 4 of 12 fibroadenomas. This morphologic characteristic was seen only in fibroadenomas and not found in other benign or malignant lesions. Similar finding was also documented in the studies by Orel \& Nunes et al 7,9,10. However, Nunes et al. reported presence of nonenhancing internal septations in an irregular and spiculated mass did not reflect benignity. Three septated irregular masses in his study were confirmed histopathologically as intraductal papillary carcinoma, colloid carcinoma with DCIS and fibrocystic change 11 .

In our study, all benign and malignant lesions were identified as low or intermediate signal on T1-weighted images and high signal on STIR sequence. The signal intensity on T2weighted images was variable. Eighteen $(78.3 \%)$ of 23 benign lesions demonstrated high signal intensity and 5 exhibited either low or intermediate signal intensity on T2weighted images. A large proportion of malignant lesions $(64.3 \%)$ were highly intense and $5(35.7 \%)$ exhibited low signal on T2-weighted images. The reason for the variability of signal intensity on T2-weighted images was not entirely clear. The lesions that had low signal intensity on both T1- and T2-weighted images were difficult to detect for precontrast tumour localization. In general, lesions in fatty involution breast were easier to be identified. Statistical analysis revealed significant correlation between different signal intensity on T2-weighted images and cytology/ histopathology results. This finding contradicted to a report by Liberman L et al. where he has concluded that signal intensity on T2WI was not a significant predictor for malignancy 12. Buadu at el. also documented unenhanced MR imaging was inadequate for lesion detection and characterisation13. All malignant lesions demonstrated either heterogeneous $(92.9 \%)$ or $\operatorname{rim}(7.1 \%)$ enhancement pattern in this study. In contrast, benign lesions exhibited homogenous or no enhancement except one lesion revealed heterogenous enhancement. These were in agreement with many previous studies $3,7,14$. However, majority of the malignant lesions were infiltrating ductal carcinoma and further study is needed to assess the enhancement pattern in lobular carcinoma, invasive lobular carcinoma and DCIS.

There was significant difference in the dynamic enhancement as represented by the time-signal intensity curves for benign and malignant enhancing lesions. In breast cancers, plateau $(14.3 \%)$ or washout $(78.6 \%)$ time course 
prevail. In contrast, benign enhancing lesions exhibited steadily progressive signal intensity time courses. Other studies have revealed the similar findings 6 . However, some researchers found that there were overlapping in enhancement between benign and malignant lesions 7,13 , 15. The rate of contrast enhancement may be related to the degree of vascularisation of a tumour than to malignancy or benignity. This was supported by the fact that malignant lesions had higher vessel densities than benign lesions 13,16 .

Review articles showed several empiric measurements of enhancement had been used by other studies. Kaiser and Zeitler has classified a lesion as suspicious if the lesion demonstrated a $100 \%$ increase in signal intensity within first two minutes after contrast agent injection 15 . Gilles et al. defined enhancement concomitant with early vascular enhancement as suggestive 17. Boetes et al. defined enhancement within 11.5 seconds after aortic enhancement as suggestive 18 . Kuhl et al. classified a lesion as suspicious if it demonstrated a greater than $60 \%$ increase in signal intensity that was apparent on the first contrast-enhanced images 6,19. Kuhl et. al. achieved a lower specificity of $37 \%$ when they used enhancement rate alone as the criterion as compared to the shape of the signal intensity time course curve where they achieved specificity of $83 \% 6$.

It is becoming increasingly clear that while most investigators have used either enhancement kinetics or morphological appearances in an attempt to differentiate benign enhancing lesions from enhancing breast cancers, an integrated interpretation strategy where the combined enhancement kinetics data and morphologic feature analysis may be superior than the use of either method alone 20,21. Malignant lesions identified in this study, were mainly infiltrating ductal carcinoma confirmed by cytology or histopathology. Due to small sample size, no DCIS was found in this study. Thus the sensitivity of MRI in detecting DCIS in this study cannot be evaluated.

Wurdinger et al. had reported 5 of 41 carcinoma in situ were missed even after re-evaluation 22. This was attributed to none or late contrast enhancement. Kaiser et al. reported $20 \%$ of lesions were detected only because of the tumour enhancement 15 . Among 36 women with DCIS, Gilles et al. demonstrated 2 cases with comedo-type without early contrast enhancement 23. Orel et al. also documented 3 cases of DCIS were not identified on MR imaging as there was neither microcalcifications nor abnormal enhancement 7. Liberman et.al. reported a washout kinetic pattern in $70 \%$ of infiltrating carcinoma but only in $9 \%$ of DCIS lesions. The lower frequency of washout in DCIS may attribute to differences in vascularity between in situ and invasive cancers. They identified most malignant masses were infiltrating carcinoma, whereas most malignant non mass lesions were DCIS 12.

Neubauer et al. studied on the high grade and non-high grade DCIS and found that unilateral segmental enhancement to be the most commonly seen and most striking feature of DCIS $(82 \%)$, usually combined with a granular dotted pattern, M2 (73\%). However, linear or linear-branched enhancement was not found in his study. He concluded that a normal MR imaging may exclude high grade DCIS 24. Secondary signs of carcinoma were well identified on MR imaging. The enhancement characteristics of the nipple and retroareolar region were considered abnormal when the signal intensity of the nipple and retroareolar region exceeded that of the normal breast parenchyma following intravenous contrast administration5. We observed the involvement of skin, retroareolar, nipple and pectoralis muscle were demonstrated exclusively in the malignant lesions only. Therefore presence of these structures involvement suggestive of a malignant breast lesion. These are important for preoperative evaluation of breast cancer, especially for posterior and retroareolar located breast cancer. Having said that, malignant lesions can not virtually be excluded if there is absence of such findings.

Several studies have demonstrated that MR imaging can offer more accurate determination of tumour extent $5,25,26$. Potential clinical indications include staging newly diagnosed breast cancer before excisional biopsy, identifying the extent of residual disease following excisional biopsy where the tumour is identified at the margin of resection and identifying a mammographically and clinically occult primary breast cancer in patients presenting with metastatic axillary node from an unknown primary 27. Conrad et al. reported that MR mammography changed the surgical procedure offered in more than half of the patients (19 of 37) 28. The sensitivity, specificity, PPV, NPV and accuracy were very high for each evaluated variables in this study. The sensitivity ranged from 85.7 to $100 \%$; specificity 95.6 - $100 \%$; PPV $93.3-100 \%$; NPV 92.0 - 100\%; accuracy $94.6-97.3 \%$ for the criteria evaluated. This could be due to the patient selection criteria where only cytology/histopathology proven breast masses, either benign or malignant, were included in our study. The presented malignancies were mainly infiltrating ductal carcinomas. Chronic granulomatous mastitis and lactational changes were excluded from this study. Patient referral biasness might also contribute to these favourable results.

Review of the literature yields a wide range of results, with reported specificities ranging from $37 \%$ to $97 \%$ 
3,15,18,29,30. Although MR imaging has demonstrated variable specificity, the demonstration of invasive breast cancer has approached $100 \%$ in several series 3,7,15,17,18. The invasive cancers in these studies have predominantly been invasive ductal carcinoma. In terms of the sensitivity of MR imaging for detection of invasive lobular carcinoma, relatively few studies has been published 31,32,33. Weinstein et al.described a spiculated or irregular mass, regional or multifocal contrast enhancement and regional enhancement with architectural distortion on MR imaging in 18 women with infiltrating lobular carcinoma 32. Qayyum et al. found 3 basic patterns of infiltrating lobular carcinoma on MR imaging. The commonest pattern was of multiple enhancing foci with either connecting enhancing strands or non enhancing intervening tissue. The other patterns were a solitary mass with irregular margin and enhancing septa only 33. Although MRI appears to be highly sensitive for the detection of breast cancer, the actual false negative rate of MR imaging is currently unknown. There have been reports of false negative cases on MR imaging, including cases of invasive lobular carcinoma, tubular carcinoma and DCIS 3,17,22,34.

\section{Conclusion}

A combination of lesion morphology and enhancement characteristics is useful in distinguishing benign from malignant lesions. The significant MR variables were lesion margin and shape, enhancement patterns and time-signal intensity curves. The poorly defined and spiculated margin, irregular shape, heterogeneous and rim enhancement, plateau (type II) and washout (type III) time-signal intensity curves were associated with malignancy. In contrast, well-defined border, regular shape, non or homogenous enhancement and type I time-signal intensity curve were highly suggestive of benign lesions. Internal septation was associated with fibroadenoma. MR imaging is a valuable complementary breast imaging tool to further evaluate equivocal findings on conventional x-ray mammography.

\section{References}

1. Page D.L. \& Winfield A.C. The dense mammogram. AJR 1986;147: 487-489.

2. Boetes C., Mus R.D.M., Holland R., Barentsz J.O., Strijk S.P., Wobbes T., Hendriks J.H. C.L., Ruys S.H.J. Breast tumours: comparative accuracy of MR imaging relative to mammography and US in demonstrating extent. Radiology 1995;197:743-747.

3. Harms S.E., Flamig D.P., hesley K.L., Meiches M.D., Jensen R.A., Evans W.P., Savino D.A. \& Wells R.V. MR imaging of the breast with rotating delivery of excitation off resonance: clinical experience with pathologic correlation. Radiology1993;187: 493- 501.

4. Orel S.G., Schnall M.D., Powell C.M.,hochman M.G., Solin L.J., Fowble B. L., Torosian M.H. \& Rosato E.F. Staging of suspected breast cancer: Effect of MR imaging and MR-guided biopsy. Radiology 1995;196: 115-122

5. Mumtaz H., Hall-Craggs M.A., Davidson T., Walmsley K., Thurell W., Kissin M.W. \& Taylor I. Staging of symptomatic primary breast cancer with MR imaging. AJR 1997;169: 417-424.

6. Kuhl C.K., Mielcareck P., Klaschick S., Leutner C., Wardelmann E., Gieselce J. Dynamic breast MR imaging: are signal intensity time course data useful for differential diagnosis of enhancing lesion? Radiology 1999;211: 101-10.

7. Orel S.G., Schnall M.D. \& LiVolsi V.A. Suspicious breast lesions: MR imaging with radiologicpathologic correlation. Radiology 1994;190: 485-93.

8. Nunes L.W., Schanll M.D., Orel S.G., Hochman M.G., Langlotz C.P., Reynolds C.A. \& Torosian M.H. Breast MR imaging: interpretation model. Radiology 1997; 202: 833-841.

9. Nunes L.W., SchnallM.D., Siegelman E.S., Langlotz C.P., Orel S.G., Sullivan D., Muenz L.A. Reynolds C.A. \& Torosian M.H. Diagnostic performance characteristics of architectural features revealed by high spatialresolution MR imaging of the breast. AJR 1997;169: 409-415.

10. Nunes L.W., Schnall M.D., Orel S.G., Hochman M.G., Langlotz C.P., Reynolds C.A. \& Torosian M.H. Correlation of lesion appearance and histologic findings for the nodes of a breast MR imaging interpretation model. Radiographics 1999; 19:79-92.

11. Nunes L.W., Schnall M.D. \& Orel S.G. Update of breast MR imaging Architectural Interpretation Model. Radiology 2001;219: 484-494.

12. Liberman L., Morris E.A., Joo-Young Lee M., Kaplan J.B., LaTrenta L.R., Menell J.H., Abramson A.F., Dashnaw S.M., Balion D.J. \& Dershaw D.D. Breast lesion detected on MR imaging: features and positive predictive value. AJR 2002;179: 171-178.

13. Buadu L.D., Murakami J., Murayama S., Hashiguchi N., Sakai S., Toyoshima S., Kuroki S.\& Ohmo S. Breast lesions: correlation of 
contrast medium enhancement patterns on MR images with histopathologic findings and tumour angiogenesis. Radiology 1996;200: 639-649.

14. Brinck U., Fisher U., Korabiowska M., Jutrowski M., Schauer A. \& Grabbe E. The variability of fibroadenoma in contrast-enhanced dynamic MR mammography. AJR 1997;168: 1331-1334.

15. Kaiser W.A. \& Zeitler E. MR imaging of the breast: Fast imaging sequences with and without Gd-DTPA preliminary observations. Radiology 1989;170:681686.

16. Hulka C.A., Smith B.L., Sgroi D.C., Tan L., Edmister W.B., Semple J.P., Campbell T., Kopans D.B., Brady T.J. \& Weisskoff R.M. Benign and malignant breast lesions: differentiation with Echo-planar MR imaging. Radiology 1995;197: 3338.

17. GillesR.,GuinebretiereJ-M., Lucidarme O., Philippe C., Janaud G., Finet J-F., Tardivon A.A., Masselot J. \& Vanel D. Nonpalpable breast tumours: diagnosis with contrast-enhanced subtraction dynamic MR imaging. Radiology 1994;191: 625-631.

18. Boetes C., Barentz J. J. \& Mus R.D., Sluis R.F., Erning L.T.O.,Hendriks J.H.C.L., Hollands R. \& Ruys S.H.R. MR characterization of breast lesions with a gadolinium-enhanced TurboFLASH subtraction technique. Radiology 1994;193: 777-781.

19. Kuhl C.K., Bieling H.B., Gieseke J., Kreft B.P., Sommer T., Lutterbey G. \& Schild H.H. Healthy premenopausal breast parenchyma in dynamic contrast enhanced MR imaging of the breast: normal contrast medium enhancement and cyclicalphase dependency. Radiology 1997;203: 137-144.

20. Liu P.F., Debatin J.F., Caduff R.F., Kacl G., Garzoli E. \& Krestin G.P. Improved diagnostic accurary in dynamic contrast enhanced MRI of the breast by combined quantitative and qualitative analysis. BJR 1998;71: 501-509.

21. Kinkel K., Helbich T.H., Esserman L.J., Barclay J., Schwerin E.H., Sickles E.A. \& Hylton N.M. Dynamic high-spatial-resolution MR imaging of suspicious breast lesions-diagnostic criteria and interobserver variability. AJR 2000;175: 35-43.

22. Wurdinger S., Kamprath S. Eschrich D. Schneider A and Kaiser W.A. False- negative findings of malignant breast lesions on preoperative magnetic resonance mammography. The breast 2001;10: 131-39.
23. Gilles R., Zafrani B., Guinebretiere J-M., Meunier M., Lucidarme O., Tardivon A.A.,Rochard F., vanel D., neuenschwander S.\& Arriagada R. Ductal carcinoma in situ: MR imaginghistopathologic correlation. Radiology 1995;196: 415-419.

24. Neubauer H., Li M., Kuehne-Heid R., Schneider A. \& Kaiser W.A. High grade and non-high grade ductal carcinoma in situ on dynamic MR mammography: characteristic findings for signal increase and morphological patterns of enhancement. BJR 2003;76: 3-12.

25. Morris E.A., Schwartz L.H., Drotman M.B., Kim S.J., Tan L.K. Liberman L., Abramson A.F., Van Zee K.J. \& Dershaw D.D. Evaluation of pectoralis major muscle in patients with posterior breast tumours on breast MR imaging: early experience. Radiology 2000;214: 67-72.

26. Rieber A., Schirrmeister H.,Gabelmann A., Nuessle K., Reske S., Kreienberg R., Brambs H.J. \& Kuehn T. Pre-operative staging of invasive breast cancer with MR mammography and/or PET: boon or bunk? BJR 2002;75: 789 - 798 .

27. Morris E.A., Schwartz L.H., Dershaw D.D., Van Zee K.J., Abramson A.F. \& Liberman L. MR imaging of the breast in patients with occult primary breast carcinoma. Radiology 1997;205: 437-440.

28. Conrad C., Corfitsen M.T., Gyldholm N. \& Christiansen F.L. Pre-operative MRmammography in breast cancer patients. European Journal of Surgical Oncology 1999;25: 142 - 145.

29. Heywang S.H., Wolf A., Pruss E., Hilbertz ., Eiermann W. \& Permanetter W. MR imaging of the breast with Gd-DTPA: use and limitations. Radiology 1989;171: 95-103.

30. Hulka C.A., Smith B.L., Sgroi D.C., Tan L., Edmister W.B., Semple J.P., Campbell T., Kopans D.B., Brady T.J. \& Weisskoff R.M. Benign and malignant breast lesions: differentiation with Echo-planar MR imaging. Radiology 1995;197: 33-38.

31. Rodenko G.N., harms S.E.,Pruneda J.M., Farrell R.S., Evans W.P., Copit D.S.,krakos P.A. \& Flamig D.P. MR imaging in the management before surgery of lobular carcinoma of the breast: correlation with pathology. AJR 1996;167: 14151419.

32. Weinstein S.P.,Orel S.G., Heller R., Reynolds C., 
Czerniecki B., Solin L.J. \& Schnall M. MR imaging of the breast in patients with invasive lobular carcinoma. AJR 2001;176: 399-406.

33. Qayyum A., Birdwell R.L., Daniel B.L., Nowels K.W., Jeffrey S.S., Agoston T.A.\& Herfkens R.J. MR imaging features of infiltrating lobular carcinoma of the breast-histopathologic correlation. AJR 2002;178: 1227-1232.

34. Orel S.G., Mendonca M.H., Reynolds C., Schnall M.D., Solin L.J. \& Sullivan D.C. R imaging of ductal carcinoma in situ. Radiology 1997;202: 413-420. 